\title{
Systematic review of saturated fatty acids on inflammation and circulating levels of adipokines
}

\author{
Susana Santos ${ }^{a, *}$, Andreia Oliveira ${ }^{a, b}$, Carla Lopes ${ }^{a, b}$ \\ a Institute of Public Health, University of Porto, Porto, Portugal \\ ${ }^{\mathrm{b}}$ Department of Clinical Epidemiology, Predictive Medicine and Public Health and Cardiovascular Research \& Development Unit, \\ University of Porto Medical School, Porto, Portugal
}

\section{A R T I C L E I N F O}

Article history:

Received 26 November 2012

Revised 19 June 2013

Accepted 1 July 2013

Keywords:

Saturated fatty acids

Human

Inflammation

Adipokines

C-reactive protein

\begin{abstract}
A B S T R A C T
Diet is one factor that plays a part in coronary heart disease risk through multiple biological mechanisms including subclinical inflammation. In this review, we aimed to systematically assess and summarize evidence regarding the association of saturated fatty acids (SFAs) with inflammatory markers and adipokines. An electronic search of the literature was conducted up to September 2010 using Medline, Scopus, Web of Science, and Science Direct (updated from September 2010 to August 2011 through Medline). Original studies that were written in Portuguese, English, Spanish, or French, and addressed the effects of SFA (not dietary sources or SFA-rich diets) on inflammatory markers or adipokines in adult populations were considered eligible. Data from 15 studies providing adjusted estimates were extracted. The publication year varied from 1995 to 2010 and the sample size from 54 to 4900. Most studies were cross sectional, with 3 studies using a prospective design. Twelve studies assessed total SFA, and 3 studies considered their subtypes, which were measured through dietary assessments (11 studies) or in blood samples (4 studies). Significant positive associations were observed between SFA and soluble intercellular adhesion molecule-1 and interleukin-6, whereas no significant associations were observed with E-selectin, tumor necrosis factor $\alpha$, granulocyte-macrophage colony-stimulating factor, fibrinogen, and adiponectin. For high-sensitivity C-reactive protein, 2 studies showed significant positive associations, whereas 3 studies reported no significant associations. One study reported a significant inverse association of SFA with leptin, although the other 3 found no significant associations. Based on this systematic review, a potential positive association of SFA with high-sensitivity C-reactive protein but not with adipokines is suggested, which should be confirmed by future research.
\end{abstract}

(c) 2013 Elsevier Inc. All rights reserved.

Abbreviations: BMI, body mass index; CHD, coronary heart disease; CVD, cardiovascular diseases; FFQ food frequency questionnaires; GM-CSF, granulocyte-macrophage colony-stimulating factor; hs-CRP, high-sensitivity C-reactive protein; IL-6, interleukin-6; PPAR- $\gamma$, peroxisome proliferator-activated receptor- $\gamma$; SFA, saturated fatty acids; sICAM-1, soluble intercellular adhesion molecule-1; sVCAM-1, soluble vascular cellular adhesion molecule-1; TNF- $\alpha$, tumor necrosis factor $\alpha$; US, United States.

* Corresponding author. Institute of Public Health, University of Porto, Rua das Taipas, 135, 4050-600 Porto, Portugal. Tel.: +351 222 061820 ; fax: +351 222061821.

E-mail address: susana.santos@ispup.up.pt (S. Santos). 


\section{Introduction}

Long-standing public health recommendations emphasize the importance of reducing saturated fatty acid (SFA) intake as a means of preventing cardiovascular diseases (CVDs), namely, coronary heart disease (CHD) [1]. The relationship of these fatty acids with CHD risk has traditionally been explained through their well-established effects on lipids and lipoproteins [2]. Nevertheless, the effects of diet on CHD seem to be mediated through multiple biological mechanisms that include oxidative stress, endothelial dysfunction, insulin sensitivity, blood pressure, thrombotic tendency, and subclinical inflammation [3].

Several inflammatory markers have been suggested as potentially useful predictors of prevalent or incident CVD, such as cell adhesion molecules, cytokines, acute-phase proteins, and white blood cell count [4]. According to metaanalyses of long-term prospective studies, positive associations with CHD risk have been reported for fibrinogen $[5,6]$, high-sensitivity C-reactive protein (hs-CRP) [7], white blood cell count [6], and interleukin-6 (IL-6) [8], whereas no associations have been found with soluble cell adhesion molecules [9]. A number of adipokines-proteins produced mainly by adipocytes [10]—are linked to inflammation and probably to CHD also. Of these, increasing attention has been paid to the antiinflammatory and proinflammatory effects of adiponectin and leptin, respectively [10]. Meta-analyses of prospective studies suggested inverse associations (for adiponectin) and positive associations (for leptin) with CHD, but these associations were more modest than previously suspected [11,12].

Thus far, no systematic evidence on the relationship between SFA and inflammatory outcomes has been put forward. Therefore, by systematically reviewing and summarizing evidence that addressed the effects of SFA on inflammatory markers and adipokines, we aimed to understand if SFA could influence CHD risk through an inflammatory pathway.

\section{Methods}

\subsection{Search strategy}

Potentially eligible studies were identified by an electronic search through Medline, Scopus, Web of Science, and Science Direct databases using a search expression to identify the exposure ("saturated fatty acids" OR "saturated fat") combined with a search expression to identify the outcomes of interest ("inflammation" OR "cell adhesion molecules" OR "cytokines" OR "interleukins" OR "tumor necrosis factor-alpha" OR "fibrinogen" OR "serum amyloid A protein" OR "C-reactive protein" OR "leukocyte count" OR "inflammation mediators" OR "adipokines" OR "adiponectin" OR "resistin" OR "leptin"). To optimize the search strategy, the syntax was adapted for each electronic database used, and when applicable, the terms were searched as indexed and as free-text terms. A search filter was applied to the Medline syntax (NOT (animals [mh] NOT humans [mh])) to retrieve only human studies. The literature search was conducted until August 2011. Firstly, the electronic databases were searched from inception up to September 2010. After deleting duplicates, the initial search yielded 1066 potentially eligible studies, which were screened in a 2-stage process (based on the reading of titles and/or abstracts and then of full text). To standardize procedures, this 2-stage screening process was independently conducted by 3 researchers in a subsample of 30 studies. Discrepancies in the evaluation of the studies were resolved by unanimous agreement.

This search was supplemented by hand searching the reference lists of original studies and reviews. A search update was conducted in August 2011 through Medline database (because $95 \%$ of the eligible studies identified in the main search were retrieved from Medline), yielding 51 additional potentially eligible studies that were also screened in the 2stage process that was previously described.

\subsection{Exclusion criteria}

The following exclusion criteria were set a priori and considered in the 2-stage screening process: (1) nonoriginal studies, (2) studies with data unrelated to the effects of SFA on inflammatory markers or adipokines, (3) nonhuman/in vitro studies, (4) nonadult population studies ( $<17$ years old), (5) studies that did not evaluate SFA but rather their dietary sources or SFA-rich diets, (6) studies that did not provide the association's estimates of SFA with inflammatory markers or adipokines, and (7) studies that were not written in Portuguese, English, Spanish, or French. No studies were excluded because of weakness of design or lack of data quality.

\subsection{Data extraction}

Eligible studies were reviewed, and data were extracted using a predefined extraction form. Disagreement in the studies' evaluation was resolved through consensus, which involved a discussion among researchers. From each study, the following information was extracted: first author, publication year, country of origin, study design/analysis approach, sample characteristics (size, sex distribution, age, and, when applicable, a general description of participants), type and assessment method of the exposure, outcome, covariates, and results. Only adjusted estimates were extracted. When a study presented different adjustment models, we extracted estimates from the model considered by the authors as the final one. If this criterion could not be applied, estimates adjusted for the largest number of possible confounding variables were considered.

\section{Findings of SFA on inflammation and circulating levels of adipokines}

Twenty-three studies were considered eligible, of which 21 studies were identified through both electronic searches conducted [13-33] and 2 studies through the reference lists searched $[34,35]$. Of these, 15 studies presented adjusted estimates, and therefore, data were extracted [13-26,35] (Fig.). The main characteristics of these studies and the results on the associations between SFA and inflammatory markers or adipokines are summarized in Tables 1-4. Studies were grouped by type of outcome: cell adhesion molecules (Table 1), cytokines (Table 2), acute-phase proteins (Table 3), and adipokines (Table 4). 


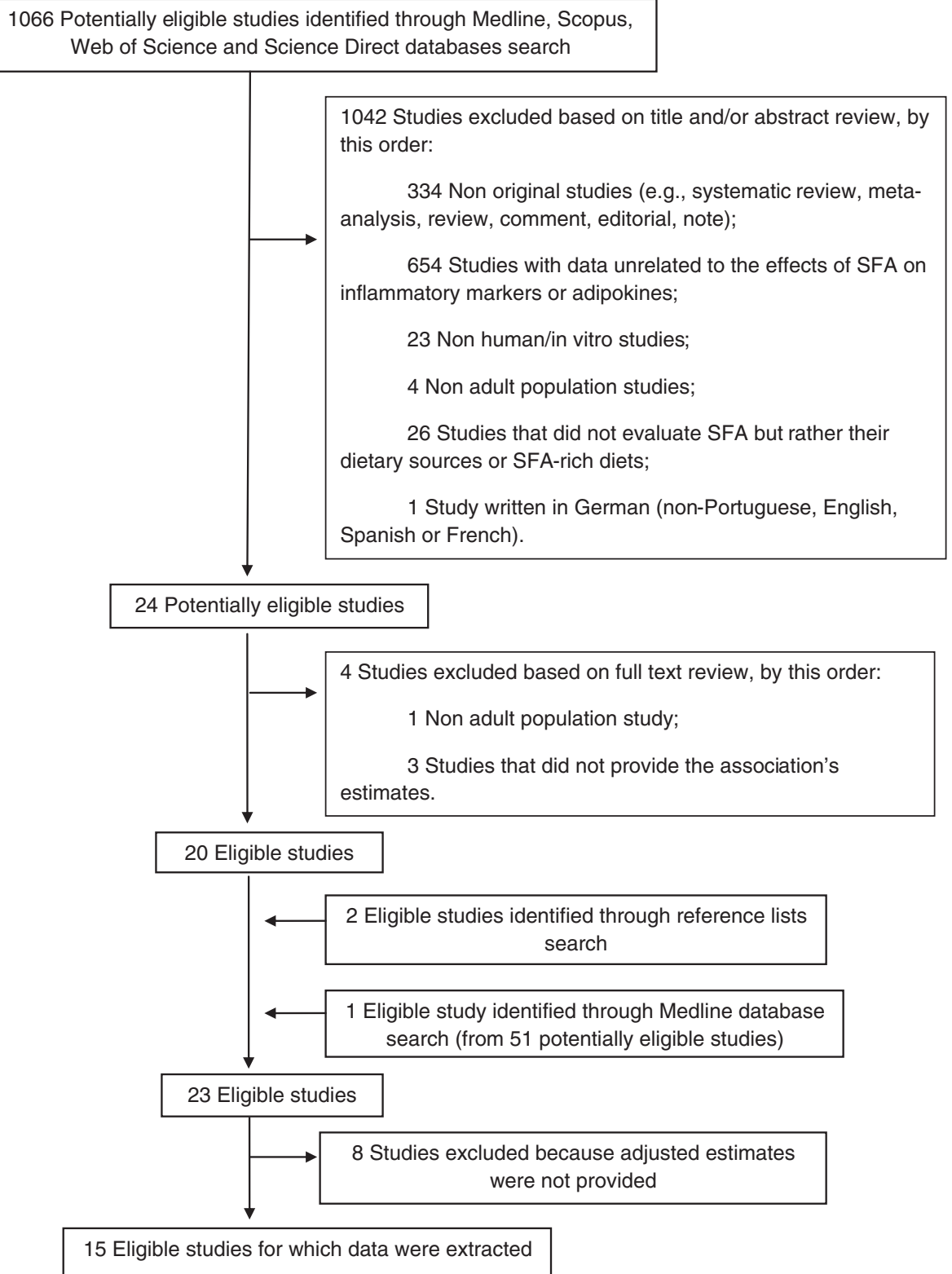

Fig. - Systematic review flowchart.

The publication year ranged from 1995 to 2010. There were 6 studies conducted in North America [13,16,17,20,21,35], 7 in Europe $[14,15,18,19,24-26]$, and 2 in Japan [22,23]. Most studies were cross sectional $[13,16-20,22-26,35]$, with 3 studies using a prospective design $[14,15,21]$. The number of participants in each study ranged from 54 to 4900 . There were 4 studies conducted exclusively in men $[16,17,24,35], 3$ studies conducted exclusively in women $[20,22,23]$, and 8 studies that included both sexes [13-15,18,19,21,25,26]. The effects on inflammatory markers and adipokines were assessed in 12 studies for total SFA [13-17,19-24,35] and in 3 studies for their subtypes $[18,25,26]$. Dietary assessments were used in 11 studies and included 24-hour recalls [13], food frequency questionnaires (FFQs) [14-16,20,21,35], food records [17], and dietary histories [22-24]. The other 4 studies measured fatty acids in blood samples $[18,19,25,26]$. The statistical analysis conducted, as well as the level of adjustment performed, varied across studies.

\subsection{Cell adhesion molecules}

Three studies on SFA and cell adhesion molecules were included and focused on soluble intercellular adhesion molecule-1 (sICAM-1), soluble vascular cellular adhesion molecule-1 (sVCAM-1), and E-selectin (Table 1). There was a significant trend between quintiles of change in the percentage of energy intake from SFA and changes in SICAM-1 concentration after 2 years of follow-up (P for trend $<.001$ ) in moderately hypercholesterolaemic Dutch subjects with at least 2 of the following cardiovascular risk factors: high blood pressure (diastolic $\geq 95 \mathrm{~mm} \mathrm{Hg}$ and/or systolic $\geq 160 \mathrm{~mm} \mathrm{Hg}$ ) or 
Table 1 - Studies on SFAs and cell adhesion molecules included in this systematic review

\begin{tabular}{|c|c|c|c|c|c|c|c|}
\hline \multirow{2}{*}{$\begin{array}{l}\text { First author, } \\
\text { publication } \\
\text { year, country } \\
\text { of origin }\end{array}$} & \multirow{2}{*}{$\begin{array}{l}\text { Study } \\
\text { design/ } \\
\text { analysis } \\
\text { approach }\end{array}$} & \multirow{2}{*}{$\begin{array}{c}\text { Sample } \\
\text { characteristics }\end{array}$} & \multicolumn{2}{|c|}{ Exposure } & \multirow[t]{2}{*}{ Outcome } & \multirow[t]{2}{*}{ Covariates } & \multirow[t]{2}{*}{ Results } \\
\hline & & & Type & $\begin{array}{l}\text { Assessment } \\
\text { method }\end{array}$ & & & \\
\hline $\begin{array}{l}\text { Bemelmans } \\
\text { [14], 2002, } \\
\text { the Netherlands }\end{array}$ & $\begin{array}{l}\text { Before- } \\
\text { and-after } \\
\text { analysis }\end{array}$ & $\begin{array}{l}103 \text { ( } 37 \% \text { men); } \\
\text { age range, } 30-70 \\
\text { y; subjects with } \\
\text { elevated risk at } \\
\text { developing } \\
\text { CVDs }\end{array}$ & SFA & $\begin{array}{l}\text { Semiquantitative } \\
\text { FFQ (reference } \\
\text { period unknown) }\end{array}$ & sICAM-1 & $\begin{array}{l}\text { Baseline sICAM-1 } \\
\text { concentration, BMI and } \\
\text { SFA intake, age and } \\
\text { changes in intake of fruit, } \\
\text { polyunsaturated fat, and } \\
\text { dietary cholesterol }\end{array}$ & $\begin{array}{l}\text { Mean (SEM) of } \\
\text { changes in sICAM-1 } \\
\text { concentration per } \\
\text { quintile of change in } \\
\text { the \% of energy intake } \\
\text { from SFA (mean [SD]) } \\
\text { after } 2 \text { y } \\
\text { Q1 (-5.9 [1.4]): } \\
-19.0(5.6) \\
\text { Q2 }(-2.7[0.4]): \\
-12.7(4.9) \\
\text { Q3 }(-1.7[0.3]): \\
-2.0(4.8) \\
\text { Q4 }(-0.3[0.5]): 5.6(5.0) \\
\text { Q5 }(1.6[0.7]): 8.6(5.3) \\
\text { P for trend < } 001 ; \\
\text { n = } 78\end{array}$ \\
\hline $\begin{array}{l}\text { Couillard [17], } \\
\text { 2006, Canada }\end{array}$ & $\begin{array}{l}\text { Cross } \\
\text { sectional }\end{array}$ & $\begin{array}{l}54 \text { men; mean } \\
\text { (SD) age, } 40 \text { y } \\
\text { (12); apparently } \\
\text { healthy but } \\
\text { abdominally } \\
\text { obese }\end{array}$ & SFA & $\begin{array}{l}\text { 3-d food record ( } 2 \\
\text { weekdays and } 1 \\
\text { weekend day) }\end{array}$ & $\begin{array}{l}\text { sICAM-1, } \\
\text { sVCAM-1 }\end{array}$ & $\begin{array}{l}\text { Age, BMI, waist-to-hip } \\
\text { ratio, abdominal visceral } \\
\text { adipose tissue area, } \\
\text { dietary monounsaturated } \\
\text { and polyunsaturated fat } \\
\text { intakes, cholesterol, total } \\
\text { daily energy, } \\
\text { carbohydrate intakes }\end{array}$ & $\begin{array}{l}\text { Partial }\left(R^{2 *} 100\right) ; \\
\text { P value: } \\
\text { sICAM-1 8.87; .0320 } \\
\text { sVCAM-1 8.82; .0325 }\end{array}$ \\
\hline $\begin{array}{l}\text { Petersson [25], } \\
\text { 2009, Sweden }\end{array}$ & $\begin{array}{l}\text { Cross } \\
\text { sectional }\end{array}$ & $\begin{array}{l}264 \text { ( } 56 \% \text { men); } \\
\text { age, } 70 \text { y }\end{array}$ & $\begin{array}{l}\text { Myristic } \\
\text { acid } \\
(14: 0) ; \\
\text { palmitic } \\
\text { acid } \\
(16: 0) ; \\
\text { stearic } \\
\text { acid (18:0) }\end{array}$ & $\begin{array}{l}\text { Measured in } \\
\text { serum } \\
\text { cholesteryl } \\
\text { esters }\end{array}$ & E-selectin & $\begin{array}{l}\text { BMI, smoking habits, } \\
\text { alcohol consumption, } \\
\text { physical activity, use of } \\
\text { lipid lowering drugs }\end{array}$ & $\begin{array}{l}\text { P value: } \\
\text { Myristic acid: } .77 \\
\text { Palmitic acid: } .54 \\
\text { Stearic acid: } .31 \\
\mathrm{n}=211\end{array}$ \\
\hline
\end{tabular}

use of antihypertensive medication, body mass index (BMI) $\geq 27 \mathrm{~kg} / \mathrm{m}^{2}$, smoking, history of CVD, and/or family history of onset of CVD before the age of 60 years. In the first quintile (corresponding to a decrease of 5.9\% of energy intake from SFA), the sICAM-1 concentration decreased $19.0 \mathrm{ng} / \mathrm{mL}(\mathrm{SEM}=5.6)$ compared with an increase of $8.6 \mathrm{ng} / \mathrm{mL}(\mathrm{SEM}=5.3)$ in the fifth quintile (corresponding to an increase of $1.6 \%$ of energy intake from SFA) [14]. In another study [17], the intake of SFA was the best predictor of the variance in circulating SICAM-1 $(P=$ .0320) and sVCAM-1 $(P=.0325)$ concentrations in apparently

Table 2 - Studies on SFAs and cytokines included in this systematic review

\begin{tabular}{|c|c|c|c|c|c|c|c|}
\hline \multirow{2}{*}{$\begin{array}{l}\text { First author, } \\
\text { publication } \\
\text { year, country } \\
\text { of origin }\end{array}$} & \multirow{2}{*}{$\begin{array}{l}\text { Study } \\
\text { design/ } \\
\text { analysis } \\
\text { approach }\end{array}$} & \multirow[t]{2}{*}{ Sample characteristics } & \multicolumn{2}{|c|}{ Exposure } & \multirow[t]{2}{*}{ Outcome } & \multirow[t]{2}{*}{ Covariates } & \multirow[t]{2}{*}{ Results } \\
\hline & & & Type & $\begin{array}{c}\text { Assessment } \\
\text { method }\end{array}$ & & & \\
\hline $\begin{array}{l}\text { Kalogeropoulos } \\
\text { [19], 2010, Greece }\end{array}$ & $\begin{array}{l}\text { Cross } \\
\text { sectional }\end{array}$ & $\begin{array}{l}374 \text { ( } 51 \% \text { men); mean (SD) } \\
\text { age: men, } 44 \text { y (13); } \\
\text { women, } 40 \text { y (15); } \\
\text { apparently healthy }\end{array}$ & SFA & $\begin{array}{l}\text { Measured in } \\
\text { plasma }\end{array}$ & $\begin{array}{l}\text { IL-6; } \\
\text { TNF- } \alpha\end{array}$ & $\begin{array}{l}\text { Age, sex, smoking habits, } \\
\text { physical activity status, } \\
\text { energy intake, } \\
\text { dietary habits through } \\
\text { the MedDietScore, BMI }\end{array}$ & $\begin{array}{l}\beta \pm \mathrm{SE} \\
\text { (P value): } \\
\text { IL-6: } 0.02 \pm \\
0.008(.01) \\
\text { TNF- } \alpha: 0.17 \pm \\
0.10(.08)\end{array}$ \\
\hline $\begin{array}{l}\text { Fernández-Real } \\
\text { [18], 2001, Spain }\end{array}$ & $\begin{array}{l}\text { Cross } \\
\text { sectional }\end{array}$ & $\begin{array}{l}78 \text { ( } 49 \% \text { men); mean (SD) } \\
\text { age: men, } 40.1 \text { y (13.3); } \\
\text { women, } 38.1 \text { y }(9.3)\end{array}$ & $\begin{array}{l}\text { Palmitic } \\
\text { acid }(16: 0)\end{array}$ & $\begin{array}{l}\text { Measured in } \\
\text { serum }\end{array}$ & GM-CSF & Not specified & $\begin{array}{l}\text { In women: } \\
\beta \text { (P value): } \\
0.0011(.99)\end{array}$ \\
\hline
\end{tabular}

$\beta$, regression coefficient. 
Table 3 - Studies on SFAs and acute-phase proteins included in this systematic review

\begin{tabular}{|c|c|c|c|c|c|c|c|}
\hline \multirow{2}{*}{$\begin{array}{l}\text { First author, } \\
\text { publication } \\
\text { year, country } \\
\text { of origin }\end{array}$} & \multirow{2}{*}{$\begin{array}{l}\text { Study } \\
\text { design/ } \\
\text { analysis } \\
\text { approach }\end{array}$} & \multirow{2}{*}{$\begin{array}{c}\text { Sample } \\
\text { characteristics }\end{array}$} & \multicolumn{2}{|c|}{ Exposure } & \multirow[t]{2}{*}{ Outcome } & \multirow[t]{2}{*}{ Covariates } & \multirow[t]{2}{*}{ Results } \\
\hline & & & Type & $\begin{array}{l}\text { Assessment } \\
\text { method }\end{array}$ & & & \\
\hline $\begin{array}{l}\text { King [13], } \\
\text { 2003, USA }\end{array}$ & $\begin{array}{l}\text { Cross } \\
\text { sectional }\end{array}$ & $\begin{array}{l}4900 \text { men and } \\
\text { women; age } \\
\text { range, } \geq 17 \text { y }\end{array}$ & SFA & $24 \mathrm{~h}$ recall (1 day) & hs-CRP & $\begin{array}{l}\text { Age, race, sex, } \\
\text { medication for diabetes } \\
\text { and for lowering } \\
\text { cholesterol, smoking, } \\
\text { BMI, alcohol } \\
\text { consumption, exercise, } \\
\text { total caloric intake }\end{array}$ & $\begin{array}{l}\text { OR }(95 \% \text { CI) of } \\
\text { elevated hs-CRP } \\
\text { (>3.0mg/L) risk for } \\
\text { each quartile of SFA } \\
\text { relative to the lowest } \\
\text { quartile } \\
\text { Q3 vs Q1: } 1.58 \text { (1.02 to } \\
2.44) \\
\text { Q4 vs Q1: } 1.44 \text { (0.80 to } \\
\text { 2.58) }\end{array}$ \\
\hline $\begin{array}{l}\text { Kalogeropoulos } \\
\text { [19], 2010, Greece }\end{array}$ & $\begin{array}{l}\text { Cross } \\
\text { sectional }\end{array}$ & $\begin{array}{l}374 \text { ( } 51 \% \text { men); } \\
\text { mean (SD) age: } \\
\text { men, } 44 \text { y (13); } \\
\text { women, } 40 \text { y } \\
\text { (15); apparently } \\
\text { healthy }\end{array}$ & SFA & $\begin{array}{l}\text { Measured in } \\
\text { plasma }\end{array}$ & $\begin{array}{l}\text { hs-CRP; } \\
\text { fibrinogen }\end{array}$ & $\begin{array}{l}\text { Age, sex, smoking } \\
\text { habits, physical activity } \\
\text { status, energy intake, } \\
\text { dietary habits through } \\
\text { the MedDietScore, BMI }\end{array}$ & $\begin{array}{l}\beta \pm \mathrm{SE}(\mathrm{P} \text { value) } \\
\text { hs-CRP: } 0.11 \pm 0.04 \\
(.008) \\
\text { Fibrinogen: } 0.003 \pm \\
1.16(.99)\end{array}$ \\
\hline $\begin{array}{l}\text { Murakami [22], } \\
\text { 2008, Japan }\end{array}$ & $\begin{array}{l}\text { Cross } \\
\text { sectional }\end{array}$ & $\begin{array}{l}443 \text { women; age } \\
\text { range, 18- } 22 \text { y; } \\
\text { healthy and } \\
\text { lean; hs-CRP }<10 \\
\text { mg/L }\end{array}$ & SFA & $\begin{array}{l}\text { Diet history } \\
\text { questionnaire (1 } \\
\text { mo) }\end{array}$ & hs-CRP & $\begin{array}{l}\text { Residential block, size of } \\
\text { residential area, current } \\
\text { smoking, alcohol } \\
\text { drinking, dietary } \\
\text { supplement use, } \\
\text { physical activity, BMI }\end{array}$ & $\begin{array}{l}\text { OR ( } 95 \% \text { CI) of } \\
\text { elevated hs-CRP ( } \geq 1.0 \\
\mathrm{mg} / \mathrm{L}) \text { risk for SFA } \\
\text { intake above vs below } \\
\text { the median value } \\
(8.3 \% \text { of energy): } 1.22 \\
(0.52-2.83)\end{array}$ \\
\hline $\begin{array}{l}\text { Bo [15], 2008, } \\
\text { Italy }\end{array}$ & $\begin{array}{l}\text { Before- } \\
\text { and-after } \\
\text { analysis }\end{array}$ & $\begin{array}{l}335 \text { ( } 42 \% \text { men); } \\
\text { mean (SD) age, } \\
56 \text { y (6); subjects } \\
\text { with multiple } \\
\text { metabolic } \\
\text { abnormalities }\end{array}$ & SFA & $\begin{array}{l}\text { Semiquantitative } \\
\text { FFQ (reference } \\
\text { period unknown) }\end{array}$ & hs-CRP & Age, sex, actual BMI & $\begin{array}{l}\text { Effect of increased } \\
\text { SFA intake on } \\
\text { changes in hs-CRP } \\
\text { after } 1 \mathrm{y}: \beta(95 \% \mathrm{CI}), \mathrm{P} \\
\text { value: } 0.02(-0.001 \text { to } \\
0.04), .10\end{array}$ \\
\hline $\begin{array}{l}\text { Petersson [25], } \\
\text { 2009, Sweden }\end{array}$ & $\begin{array}{l}\text { Cross } \\
\text { sectional }\end{array}$ & $\begin{array}{l}264 \text { ( } 56 \% \text { men); } \\
\text { age, } 70 \mathrm{y} \text {; hs-CRP } \\
\leq 10 \mathrm{mg} / \mathrm{L}\end{array}$ & $\begin{array}{l}\text { Myristic } \\
\text { acid (14:0); } \\
\text { palmitic } \\
\text { acid (16:0); } \\
\text { stearic } \\
\text { acid (18:0) }\end{array}$ & $\begin{array}{l}\text { Measured in } \\
\text { serum } \\
\text { cholesteryl } \\
\text { esters }\end{array}$ & hs-CRP & $\begin{array}{l}\text { BMI, smoking habits, } \\
\text { alcohol consumption, } \\
\text { physical activity, use of } \\
\text { lipid lowering drugs }\end{array}$ & $\begin{array}{l}P \text { value }^{a}: \\
\text { Myristic acid: } .58 \\
\text { Palmitic acid: } .51 \\
\text { Stearic acid: } .02 \\
\mathrm{n}=211\end{array}$ \\
\hline $\begin{array}{l}\text { Ludwig [21], } \\
\text { 1999, USA }\end{array}$ & $\begin{array}{l}\text { Cohort } \\
\text { study }\end{array}$ & $\begin{array}{l}1453 \text { (white men } \\
\text { and women); } \\
1132 \text { (black men } \\
\text { and women) }\end{array}$ & SFA & $\begin{array}{l}\text { Quantitative FFQ } \\
\text { (1 mo) }\end{array}$ & Fibrinogen & $\begin{array}{l}\text { Sex, age, field center, } \\
\text { education, energy } \\
\text { intake, physical activity, } \\
\text { cigarette smoking, } \\
\text { alcohol intake, vitamin } \\
\text { supplement use }\end{array}$ & $\begin{array}{l}\text { Fibrinogen means } \\
(\mathrm{mg} / \mathrm{dL}) \text { according to } \\
\text { quintiles of SFA } \\
\text { For white men and } \\
\text { women: } \mathrm{Q} 1=251, \mathrm{Q} 5= \\
258 ; \mathrm{P} \text { for trend }=.18 \\
\text { For black men and } \\
\text { women: } \mathrm{Q} 1=270, \mathrm{Q} 5= \\
273 ; \mathrm{P} \text { for trend }=.34\end{array}$ \\
\hline $\begin{array}{l}\text { Pekkanen [24], } \\
\text { 1995, Finland }\end{array}$ & $\begin{array}{l}\text { Cross } \\
\text { sectional }\end{array}$ & $\begin{array}{l}198 \text { men; age } \\
\text { range: } 70-89 \text { y }\end{array}$ & SFA & $\begin{array}{l}\text { Dietary history } \\
\text { method (1 mo) }\end{array}$ & Fibrinogen & Age, dietary energy & $\begin{array}{l}\text { Partial spearman rank } \\
\text { correlation: }-0.02\end{array}$ \\
\hline
\end{tabular}

$\beta$, Regression coefficient.

${ }^{a} \mathrm{P}<.01$ was considered statistically significant to avoid type 1 errors caused by multiple testings.

healthy Canadian men without endocrine, cardiovascular, hepatic, and renal disorders and proinflammatory conditions, as well as nonsmokers, alcohol intake less than $30 \mathrm{~g} / \mathrm{d}$, and body weight changes less than $3 \mathrm{~kg}$ within 2 months before the study. As a group though, men who took part in this study were obese and characterized by an important accumulation of abdominal and visceral adipose tissue. However, in another study based on an elderly Swedish population, no significant associations between E-selectin and myristic acid $(P=.77)$, palmitic acid $(P=.54)$, or stearic acid $(P=.31)$ that were measured in serum cholesteryl esters were reported [25].

\subsection{Cytokines}

Two studies on SFA and cytokines were included and have focused on IL-6, tumor necrosis factor $\alpha$ (TNF- $\alpha$ ), and granulocyte-macrophage colony-stimulating factor (GM-CSF) (Table 2). Total plasma SFA presented a significant positive association with circulating IL-6 $(\beta=0.02, P=.01)$, whereas a 
Table 4 - Studies on SFAs and adipokines included in this systematic review

\begin{tabular}{|c|c|c|c|c|c|c|c|}
\hline \multirow{2}{*}{$\begin{array}{l}\text { First author, } \\
\text { publication } \\
\text { year, country } \\
\text { of origin }\end{array}$} & \multirow{2}{*}{$\begin{array}{l}\text { Study } \\
\text { design/ } \\
\text { analysis } \\
\text { approach }\end{array}$} & \multirow{2}{*}{$\begin{array}{c}\text { Sample } \\
\text { characteristics }\end{array}$} & \multicolumn{2}{|c|}{ Exposure } & \multirow[t]{2}{*}{ Outcome } & \multirow[t]{2}{*}{ Covariates } & \multirow[t]{2}{*}{ Results } \\
\hline & & & Type & $\begin{array}{l}\text { Assessment } \\
\text { method }\end{array}$ & & & \\
\hline $\begin{array}{l}\text { Murakami } \\
\text { [23], 2007, } \\
\text { Japan }\end{array}$ & $\begin{array}{l}\text { Cross } \\
\text { sectional }\end{array}$ & $\begin{array}{l}424 \text { women; age } \\
\text { range, } 18-22 \text { y; } \\
\text { healthy and lean }\end{array}$ & SFA & $\begin{array}{l}\text { Diet history } \\
\text { questionnaire } \\
(1 \mathrm{mo})\end{array}$ & Leptin & $\begin{array}{l}\text { Residential block, size of } \\
\text { residential area, current } \\
\text { smoking, rate of eating, } \\
\text { alcohol drinking, physical } \\
\text { activity, energy intake, BMI, } \\
\text { intakes of protein, dietary } \\
\text { fiber, monounsaturated and } \\
\text { polyunsaturated fatty acids }\end{array}$ & $\begin{array}{l}\text { Geometric } \\
\text { means (95\% } \\
\text { CI) for } \\
\text { leptin } \\
\text { according } \\
\text { to quintiles } \\
\text { of SFA: } \\
\text { Q1: 7.8 } \\
\text { (7.0-8.7) } \\
\text { Q2: 7.3 } \\
\text { (6.6-7.9) } \\
\text { Q3: 7.6 } \\
(7.0-8.3) \\
\text { Q4: 8.1 } \\
(7.5-8.9) \\
\text { Q5: 7.6 } \\
\text { (6.8-8.5) } \\
\text { P for } \\
\text { trend }=.88\end{array}$ \\
\hline $\begin{array}{l}\text { Kong [20], } \\
2009 \text {, USA }\end{array}$ & $\begin{array}{l}\text { Cross } \\
\text { sectional }\end{array}$ & $\begin{array}{l}173 \text { women; age } \\
\text { range, 50-75 y; } \\
\text { overweight to } \\
\text { obese } \\
\text { postmenopausal } \\
\text { and sedentary }\end{array}$ & SFA & $\begin{array}{l}\text { Semiquantitative } \\
\text { FFQ ( } 3 \mathrm{mo})\end{array}$ & Leptin & $\begin{array}{l}\text { Energy intake, BMI, } \\
\text { percentage body fat, age and } \\
\text { intra-abdominal fat }\end{array}$ & $\begin{array}{l}\beta \text { (P value): } \\
0.10(.12) \\
\mathrm{n}=162\end{array}$ \\
\hline $\begin{array}{l}\text { Chu [16], } \\
\text { 2001, USA }\end{array}$ & $\begin{array}{l}\text { Cross } \\
\text { sectional }\end{array}$ & $\begin{array}{l}268 \text { men; age } \\
\text { range, } 47-83 \text { y }\end{array}$ & SFA & $\begin{array}{l}\text { Semiquantitative } \\
\text { FFQ (1 y) }\end{array}$ & Leptin & $\begin{array}{l}\text { Age, cigarette smoking, } \\
\text { alcohol drinking, physical } \\
\text { activity, time since last meal, } \\
\text { BMI, total calories, total } \\
\text { protein, monounsaturated, } \\
\text { polyunsaturated and } \\
\text { cholesterol intake }\end{array}$ & $\begin{array}{l}\beta \pm \operatorname{SE}(P \\
\text { value }):- \\
0.41 \pm 0.75 \\
(.581)\end{array}$ \\
\hline $\begin{array}{l}\text { Warensjo [26], } \\
\text { 2004, Sweden }\end{array}$ & $\begin{array}{l}\text { Cross- } \\
\text { sectional } \\
\text { analysis }\end{array}$ & $\begin{array}{l}234(80 \% \text { men); } \\
\text { mean (SD) age, } \\
55 \text { y ( } 7)\end{array}$ & $\begin{array}{l}\text { Pentadecanoic } \\
\text { acid }(15: 0)+ \\
\text { heptadecanoic } \\
\text { acid }(17: 0)\end{array}$ & $\begin{array}{l}\text { Measured in } \\
\text { serum } \\
\text { phospholipids }\end{array}$ & Leptin & BMI & $\begin{array}{l}\text { Pearson } \\
\text { correlation } \\
\text { coefficient; } \\
\text { P value: - } \\
0.20 ;<.05\end{array}$ \\
\hline $\begin{array}{l}\text { Pischon [35], } \\
\text { 2005, USA }\end{array}$ & $\begin{array}{l}\text { Cross } \\
\text { sectional }\end{array}$ & $\begin{array}{l}532 \text { men; mean } \\
\text { (SD) age, } 65.2 \text { y } \\
(0.4)\end{array}$ & SFA & $\begin{array}{l}\text { Semiquantitative } \\
\text { FFQ (1 y) }\end{array}$ & Adiponectin & $\begin{array}{l}\text { Age, smoking status, history } \\
\text { of diabetes, history of } \\
\text { hypertension, alcohol } \\
\text { consumption, physical } \\
\text { activity, energy intake, } \\
\text { protein, monounsaturated } \\
\text { and polyunsaturated fat, BMI }\end{array}$ & $\begin{array}{l}\beta \pm \operatorname{SE}(P \\
\text { value }): 1.65 \\
\pm 1.17(.16)\end{array}$ \\
\hline
\end{tabular}

nonsignificant association was observed with circulating TNF- $\alpha(\beta=0.17, P=.08)$ in apparently healthy Greek adults (without any clinical evidence of CVD, any other atherosclerotic disease, cancer, or chronic viral infections) [19]. Serum palmitic acid exhibited a nonsignificant association with GMCSF $(\beta=0.0011, P=.99)$ in Spanish women; no adjusted estimates were shown in men [18].

\subsection{Acute-phase proteins}

Seven studies on SFA and acute-phase proteins were included and focused on hs-CRP and fibrinogen (Table 3). An increased likelihood of elevated hs-CRP (>3.0 mg/L) was found for the third quartile of SFA intake in United States (US) adults (odds ratio [OR], 1.58; 95\% confidence interval [CI], 1.02-2.44) [13]. For total SFA measured in plasma of the aforementioned apparently healthy Greek adults, a significant positive association was also reported with hs-CRP $(\beta=0.11, P=.008)$ [19]. On the contrary, in young healthy (without diabetes mellitus, hypertension, and CVD) and lean Japanese women, SFA intake above the median value ( $8.3 \%$ of energy) was not significantly associated with an increased likelihood of elevated hs-CRP ( $\geq 1.0$ mg/L; OR, 1.22; 95\% CI, 0.52-2.83) [22]. Similarly, in another study, an increased SFA intake was not significantly 
associated with changes in hs-CRP $(\beta=0.02, P=.10)$ in Italian subjects with multiple metabolic abnormalities (with either the metabolic syndrome or 2 components of the syndrome plus hsCRP serum values of $\geq 3.0 \mathrm{mg} / \mathrm{L}$ ) but without diabetes mellitus, CVD, chronic liver or kidney disease, and advanced cancer [15]. High-sensitivity CRP was also not significantly associated with myristic acid $(P=.58)$, palmitic acid $(P=.51)$, or stearic acid $(P=$ .02) that were measured in serum cholesteryl esters of the Swedish elderly population that was previously mentioned [25]. For fibrinogen, total plasma SFA exhibited a nonsignificant association ( $\beta=0.003, P=.99)$ [19]. Also, nonsignificant trends of fibrinogen concentration across quintiles of SFA intake were reported in white $(P$ for trend $=.18$ ) and black US adults ( $P$ for trend $=.34$ ) [21]. No correlation between SFA intake and fibrinogen was found in elderly Finnish men $(r=-0.02)$ [24].

\subsection{Adipokines}

Five studies on SFA and adipokines were included and have focused on leptin and adiponectin (Table 4). There was no significant trend between quintiles of SFA intake and leptin concentrations in the previously mentioned population of young healthy and lean Japanese women ( $P$ for trend $=.88$ ) [23]. Also, intake of SFA was not significantly associated with leptin concentrations $(\beta=0.10, P=.12)$ in a group of overweight to obese (BMI of at least $25.0 \mathrm{~kg} / \mathrm{m}^{2}$ or a BMI $\geq 24.0$ and $<25.0 \mathrm{~kg} / \mathrm{m}^{2}$ if percentage body fat $>33.0 \%$ ) postmenopausal and sedentary US women [20]. In a group of US men aged 47 to 83 years (who were free of CVD, diabetes mellitus, gastric or duodenal ulcer, liver disease, and cancerexcept nonmelanoma skin cancer), SFA intake was also not significantly associated with leptin concentrations $(\beta=-0.41$, $P=.581)$ [16]. Only the sum of pentadecanoic acid and heptadecanoic acid in serum phospholipids of a Swedish population was significantly and negatively correlated with leptin $(r=-0.20, P<.05)$, but the correlation's magnitude was poor [26]. In regard to adiponectin, a nonsignificant association with SFA intake $(\beta=1.65, P=.16)$ was found in US men [35].

\section{Discussion}

This systematic review sought to evaluate the effects of SFA on inflammatory markers and adipokines providing a summary of the available evidence on this topic. Overall, data were extracted and summarized for sICAM-1, sVCAM-1, E-selectin, IL-6, TNF- $\alpha$, GM-CSF, hs-CRP, fibrinogen, leptin, and adiponectin.

In 78 Dutch subjects with elevated risk of developing CVD, an increased intake of SFA was associated with increased concentrations of SICAM-1 [14], thus suggesting a detrimental role of SFA on endothelial function. However, in this high-risk population, the sICAM-1 concentrations might be a consequence of an asymptomatic arterial disease, therefore confounding the association with SFA intake. Also, in a group of 54 apparently healthy but abdominally obese Canadian men, SFA intake was the best predictor of the variance in circulating sICAM-1 and SVCAM-1 concentrations [17]. However, because the magnitude and direction of the associations were unknown, clear conclusions could not be drawn from these findings. In contrast, no associations were found between myristic, palmitic, or stearic acids measured in serum cholesteryl esters and E-selectin in 211 Swedish elderly men and women [25]. Nevertheless, the possibility of existing associations in other populations, namely, in those including younger subjects, cannot be excluded.

Very few studies evaluated the association between SFA and cytokines, yielding inconclusive findings. Total SFA measured in the plasma of 374 apparently healthy Greek adults were positively associated with circulating IL- 6 but not with TNF- $\alpha$ [19]. In Spanish women, no associations were observed between serum palmitic acid and GM-CSF [18]. It should be stressed that only 40 women were included in this analysis, and therefore, even if there is an association between palmitic acid and GM-CSF, statistical power may have been insufficient to allow its detection.

Studies on SFA and hs-CRP included in this systematic review have shown inconsistent findings. On one hand, intake of SFA was associated with an increased likelihood of elevated hs-CRP $(>3.0 \mathrm{mg} / \mathrm{L})$ in a study of US adults that presented the largest sample size $(n=4900)$ [13]. Likewise, total plasma SFAs were positively associated with circulating hs-CRP in the population of apparently healthy Greek adults [19]. On the other hand, SFA intake was not significantly associated with an increased likelihood of elevated hs-CRP $(\geq 1.0 \mathrm{mg} / \mathrm{L})$ in 443 young healthy and lean Japanese women. The authors suggested that the absence of association could be caused by the low base rate of elevated hs-CRP concentrations (5.6\%) [22]. The use of different cutoff values for defining elevated hs-CRP in this study of young Japanese women $(\geq 1.0 \mathrm{mg} / \mathrm{L})$ and in the previously mentioned study of US adults $(>3.0 \mathrm{mg} / \mathrm{L})$ did not allow strict comparisons between both studies. Also, in 335 Italian subjects with multiple metabolic abnormalities, increased SFA intake was not significantly associated with changes in hs-CRP. The authors hypothesized that in dysmetabolic subjects, the role of dietary factors such as SFA on inflammation could be less evident than in healthy subjects [15]. Lastly, no associations were found between levels of myristic, palmitic, or stearic acids in serum cholesteryl esters and circulating hs-CRP in a Swedish elderly population [25]. As stated for E-selectin, the associations between these SFA subtypes and hs-CRP should also be studied in younger subjects. With respect to fibrinogen, no associations were observed regarding the effects of total SFA, measured either in plasma or in diet $[19,21,24]$.

Intake of SFA was not significantly associated with leptin concentrations in 424 young healthy and lean Japanese women [23]. The narrower range of leptin concentration in this population could have contributed, at least partly, to this lack of association. Similarly, in 173 overweight to obese postmenopausal and sedentary US women [20], as well as in 268 US men aged 47 to 83 years [16], no associations were found between SFA intake and leptin concentrations. Only the sum of pentadecanoic acid and heptadecanoic acid in serum phospholipids of 234 Swedish men and women was significantly and negatively correlated with leptin; however, the magnitude of the correlation was poor [26]. It is possible that, because the study only adjusted for BMI, uncontrolled confounding may exist, and therefore, this relationship may be prone to bias. Further data from one study revealed a nonsignificant association with SFA intake and adiponectin in 532 US men [35]. 
Although no clear associations were reported in the literature, a possible association between SFA and inflammatory markers or adipokines could be supported, at least to some extent, by biological mechanisms. It seems that SFAs stimulate inflammatory signaling pathways by a process that involves toll-like receptor 4 [36] and, subsequently, nuclear factor $\kappa \mathrm{B}$, thus increasing the expression of a number of inflammatory genes [37-41]. A novel mechanism by which SFA might greatly amplify macrophages inflammation through a toll-like receptor 4-independent pathway has been proposed, which is dependent on the uptake and metabolic processing of SFA into ceramide [42]. The effects of SFA on adipokines seem to be mediated by the nuclear receptor peroxisome proliferator-activated receptor- $\gamma$ (PPAR- $\gamma$ ). Pharmacologic activation with PPAR- $\gamma$ agonists leads to increased plasma adiponectin $[43,44]$ and decreased plasma leptin concentrations [45]. Saturated fatty acids are thought to bind and inhibit PPAR- $\gamma$. In fact, it has been shown that dietary SFAs decrease the levels of arachidonic acid - the precursor for the PPAR- $\gamma$ ligands - in adipocyte plasma membrane phospholipids [46] or could be involved in a possible impairment in ligand-dependent activity of PPAR- $\gamma$ [47].

As in other systematic reviews, the conclusions reached by this study depend on other factors, for example, the comprehensive nature of the search strategy and the criteria used for inclusion or exclusion of studies and data extraction. Furthermore, the availability of relevant studies in the literature is, in part, a limiting factor.

In this review, the temporal sequence of the reported associations cannot be completely established because of the cross-sectional nature of most studies. However, reverse causality seemed unlikely to affect our conclusions because it was not expected that the exposures changed due to the outcomes and subclinical concentrations of inflammatory markers and adipokines are quite stable in time. Moreover, the statistical power may have been insufficient to allow the detection of significant associations owing to the relatively small sample size of most studies.

The differences across the specific populations investigated (eg, abdominally obese, elderly populations, and dysmetabolic subjects) could be pointed out as another limitation, thus complicating straight comparisons across the studies. Different findings might be found in other populations, particularly in healthy ones or in those that included younger subjects. The comparisons were also compromised by the different adjustments performed. However, most studies adjusted for energy intake either by the nutrient density method or by including energy intake as a covariate in the analysis.

Another drawback of this systematic review was its reliance on the accuracy of dietary assessments. Food frequency questionnaires are the method of choice in most studies, perhaps because they are inexpensive and can assess long-term diets [48]; however, this method is also subject to random and systematic errors. Only 4 studies provided a more objective measure of SFA exposure by directly measuring them in blood samples. However, when possible to compare, we did not find differences in results according to the type of method used-dietary reporting vs biomarkers.
Finally, the few studies available for review for each type of outcome precluded definite statements on the studied associations. In conclusion, this systematic review suggests a potential positive association of SFA with hs-CRP but does not support an association with adipokines. Further studies are needed in this poorly investigated field.

\section{Acknowledgment}

Funding was provided by Fundação para a Ciência e a Tecnologia, Portugal (PTDC/SAU-ESA/108315/2008).

\section{R E F E R E N C ES}

[1] Lichtenstein AH, Appel LJ, Brands M, Carnethon M, Daniels S, Franch HA, et al. Diet and lifestyle recommendations revision 2006: a scientific statement from the American Heart Association Nutrition Committee. Circulation 2006;114:82-96.

[2] Mensink RP, Katan MB. Effect of dietary fatty acids on serum lipids and lipoproteins. A meta-analysis of 27 trials. Arterioscler Thromb 1992;12:911-9.

[3] Hu FB, Willett WC. Optimal diets for prevention of coronary heart disease. JAMA 2002;288:2569-78.

[4] Pearson TA, Mensah GA, Alexander RW, Anderson JL, Cannon III RO, Criqui $\mathrm{M}$, et al. Markers of inflammation and cardiovascular disease: application to clinical and public health practice: a statement for healthcare professionals from the Centers for Disease Control and Prevention and the American Heart Association. Circulation 2003;107:499-511.

[5] Collaborative meta-analysis of prospective studies of plasma fibrinogen and cardiovascular disease. Eur J Cardiovasc Prev Rehabil 2004;11:9-17.

[6] Danesh J, Collins R, Appleby P, Peto R. Association of fibrinogen, C-reactive protein, albumin, or leukocyte count with coronary heart disease: meta-analyses of prospective studies. JAMA 1998;279:1477-82.

[7] Kaptoge S, Di Angelantonio E, Lowe G, Pepys MB, Thompson SG, Collins R, et al. C-reactive protein concentration and risk of coronary heart disease, stroke, and mortality: an individual participant meta-analysis. Lancet 2010;375:132-40.

[8] Danesh J, Kaptoge S, Mann AG, Sarwar N, Wood A, Angleman $\mathrm{SB}$, et al. Long-term interleukin- 6 levels and subsequent risk of coronary heart disease: two new prospective studies and a systematic review. PLoS Med 2008;5:e78.

[9] Malik I, Danesh J, Whincup P, Bhatia V, Papacosta O, Walker $\mathrm{M}$, et al. Soluble adhesion molecules and prediction of coronary heart disease: a prospective study and metaanalysis. Lancet 2001;358:971-6.

[10] Fantuzzi G. Adipose tissue, adipokines, and inflammation. J Allergy Clin Immunol 2005;115:911-9 [quiz 20].

[11] Sattar N, Wannamethee G, Sarwar N, Chernova J, Lawlor DA, Kelly A, et al. Leptin and coronary heart disease: prospective study and systematic review. J Am Coll Cardiol 2009;53: 167-75.

[12] Sattar N, Wannamethee G, Sarwar N, Tchernova J, Cherry L, Wallace AM, et al. Adiponectin and coronary heart disease: a prospective study and meta-analysis. Circulation 2006;114: 623-9.

[13] King DE, Egan BM, Geesey ME. Relation of dietary fat and fiber to elevation of C-reactive protein. Am J Cardiol 2003;92: 1335-9.

[14] Bemelmans WJ, Lefrandt JD, Feskens EJ, Broer J, Tervaert JW, May JF, et al. Change in saturated fat intake is associated with 
progression of carotid and femoral intima-media thickness, and with levels of soluble intercellular adhesion molecule-1. Atherosclerosis 2002;163:113-20.

[15] Bo S, Ciccone G, Guidi S, Gambino R, Durazzo M, Gentile L, et al. Diet or exercise: what is more effective in preventing or reducing metabolic alterations? Eur J Endocrinol 2008;159: 685-91.

[16] Chu NF, Stampfer MJ, Spiegelman D, Rifai N, Hotamisligil GS, Rimm EB. Dietary and lifestyle factors in relation to plasma leptin concentrations among normal weight and overweight men. Int J Obes Relat Metab Disord 2001;25:106-14.

[17] Couillard C, Pomerleau S, Ruel G, Archer WR, Bergeron J, Couture P, et al. Associations between hypertriglyceridemia, dietary fat intake, oxidative stress, and endothelial activation in men. Nutrition 2006;22:600-8.

[18] Fernandez-Real JM, Vayreda M, Casamitjana R, GonzalezHuix F, Ricart W. Circulating granulocyte-macrophage colony-stimulating factor and serum fatty acid composition in men and women. Metabolism 2001;50:1479-83.

[19] Kalogeropoulos N, Panagiotakos DB, Pitsavos C, Chrysohoou C, Rousinou G, Toutouza M, et al. Unsaturated fatty acids are inversely associated and $n-6 / n-3$ ratios are positively related to inflammation and coagulation markers in plasma of apparently healthy adults. Clin Chim Acta 2010;411:584-91.

[20] Kong A, Neuhouser ML, Xiao L, Ulrich CM, McTiernan A, Foster-Schubert KE. Higher habitual intake of dietary fat and carbohydrates are associated with lower leptin and higher ghrelin concentrations in overweight and obese postmenopausal women with elevated insulin levels. Nutr Res 2009;29: 768-76.

[21] Ludwig DS, Pereira MA, Kroenke CH, Hilner JE, Van Horn L, Slattery ML, et al. Dietary fiber, weight gain, and cardiovascular disease risk factors in young adults. JAMA 1999;282: 1539-46.

[22] Murakami K, Sasaki S, Takahashi Y, Uenishi K, Yamasaki M, Hayabuchi $\mathrm{H}$, et al. Total $\mathrm{n}-3$ polyunsaturated fatty acid intake is inversely associated with serum C-reactive protein in young Japanese women. Nutr Res 2008;28:309-14.

[23] Murakami K, Sasaki S, Takahashi Y, Uenishi K, Yamasaki M, Hayabuchi $\mathrm{H}$, et al. Nutrient and food intake in relation to serum leptin concentration among young Japanese women. Nutrition 2007;23:461-8.

[24] Pekkanen J, Mutanen M, Rasanen L, Rauramaa R, Nissinen A. Association of factor VIIc activity with dietary fat and blood lipids among elderly men-the Finnish cohorts of the Seven Countries Study. Nutr Metab Cardiovasc Dis 1995;5: 149-54.

[25] Petersson H, Lind L, Hulthe J, Elmgren A, Cederholm T, Riserus U. Relationships between serum fatty acid composition and multiple markers of inflammation and endothelial function in an elderly population. Atherosclerosis 2009;203: 298-303.

[26] Warensjo E, Jansson JH, Berglund L, Boman K, Ahren B, Weinehall L, et al. Estimated intake of milk fat is negatively associated with cardiovascular risk factors and does not increase the risk of a first acute myocardial infarction. A prospective case-control study. Br J Nutr 2004;91:635-42.

[27] Hernandez-Morante JJ, Milagro FI, Larque E, Lujan J, Martinez JA, Zamora S, et al. Relationship among adiponectin, adiponectin gene expression and fatty acids composition in morbidly obese patients. Obes Surg 2007;17:516-24.

[28] Lennie TA, Chung ML, Habash DL, Moser DK. Dietary fat intake and proinflammatory cytokine levels in patients with heart failure. J Card Fail 2005;11:613-8.

[29] Fredrikson GN, Hedblad B, Nilsson JA, Alm R, Berglund G, Nilsson J. Association between diet, lifestyle, metabolic cardiovascular risk factors, and plasma C-reactive protein levels. Metabolism 2004;53:1436-42.
[30] Merrill RM, Massey MT, Aldana SG, Greenlaw RL, Diehl HA, Salberg A. C-reactive protein levels according to physical activity and body weight for participants in the coronary health improvement project. Prev Med 2008;46:425-30.

[31] de Luis DA, Aller R, Gonzalez Sagrado M, Conde R, Izaola O, Perez Castrillon JL, et al. Serum visfatin concentrations are related to dietary intake in obese patients. Ann Nutr Metab 2010;57:265-70.

[32] Clarke R, Shipley M, Armitage J, Collins R, Harris W. Plasma phospholipid fatty acids and CHD in older men: Whitehall study of London civil servants. Br J Nutr 2009;102:279-84.

[33] Fernandez-Real JM, Vendrell J, Ricart W. Circulating adiponectin and plasma fatty acid profile. Clin Chem 2005;51:603-9.

[34] Fernandez-Real JM, Broch M, Vendrell J, Ricart W. Insulin resistance, inflammation, and serum fatty acid composition. Diabetes Care 2003;26:1362-8.

[35] Pischon T, Girman CJ, Rifai N, Hotamisligil GS, Rimm EB. Association between dietary factors and plasma adiponectin concentrations in men. Am J Clin Nutr 2005;81:780-6.

[36] Chait A, Kim F. Saturated fatty acids and inflammation: who pays the toll? Arterioscler Thromb Vasc Biol 2010;30:692-3.

[37] Ajuwon KM, Spurlock ME. Palmitate activates the NF-kappaB transcription factor and induces IL- 6 and TNFalpha expression in 3T3-L1 adipocytes. J Nutr 2005;135:1841-6.

[38] Song MJ, Kim KH, Yoon JM, Kim JB. Activation of toll-like receptor 4 is associated with insulin resistance in adipocytes. Biochem Biophys Res Commun 2006;346:739-45.

[39] Suganami T, Nishida J, Ogawa Y. A paracrine loop between adipocytes and macrophages aggravates inflammatory changes: role of free fatty acids and tumor necrosis factor alpha. Arterioscler Thromb Vasc Biol 2005;25:2062-8.

[40] Suganami T, Tanimoto-Koyama K, Nishida J, Itoh M, Yuan X, Mizuarai S, et al. Role of the toll-like receptor 4/NF-kappaB pathway in saturated fatty acid-induced inflammatory changes in the interaction between adipocytes and macrophages. Arterioscler Thromb Vasc Biol 2007;27:84-91.

[41] Yeop Han C, Kargi AY, Omer M, Chan CK, Wabitsch M, O'Brien $\mathrm{KD}$, et al. Differential effect of saturated and unsaturated free fatty acids on the generation of monocyte adhesion and chemotactic factors by adipocytes: dissociation of adipocyte hypertrophy from inflammation. Diabetes 2010;59:386-96.

[42] Schwartz EA, Zhang WY, Karnik SK, Borwege S, Anand VR, Laine PS, et al. Nutrient modification of the innate immune response: a novel mechanism by which saturated fatty acids greatly amplify monocyte inflammation. Arterioscler Thromb Vasc Biol 2010;30:802-8.

[43] Maeda N, Takahashi M, Funahashi T, Kihara S, Nishizawa H, Kishida K, et al. PPARgamma ligands increase expression and plasma concentrations of adiponectin, an adipose-derived protein. Diabetes 2001;50:2094-9.

[44] Miyazaki Y, Mahankali A, Wajcberg E, Bajaj M, Mandarino LJ, DeFronzo RA. Effect of pioglitazone on circulating adipocytokine levels and insulin sensitivity in type 2 diabetic patients. J Clin Endocrinol Metab 2004;89:4312-9.

[45] Zhang B, Graziano MP, Doebber TW, Leibowitz MD, WhiteCarrington S, Szalkowski DM, et al. Down-regulation of the expression of the obese gene by an antidiabetic thiazolidinedione in Zucker diabetic fatty rats and $\mathrm{db} / \mathrm{db}$ mice. J Biol Chem 1996;271:9455-9.

[46] Ibrahim A, Natrajan S, Ghafoorunissa R. Dietary trans-fatty acids alter adipocyte plasma membrane fatty acid composition and insulin sensitivity in rats. Metabolism 2005;54:240-6.

[47] Saravanan N, Haseeb A, Ehtesham NZ, Ghafoorunissa. Differential effects of dietary saturated and trans-fatty acids on expression of genes associated with insulin sensitivity in rat adipose tissue. Eur J Endocrinol 2005;153:159-65.

[48] Willett W. Nutritional epidemiology. 2nd ed. New York: Oxford University Press; 1998. 